\title{
HYBRID DIFFERENCE-DIFFERENTIAL BOUNDARY-VALUE PROBLEM
}

\author{
A. SAMOILENKO, A. BOICHUK, AND S. CHUIKO
}

Received 19 March, 2017

\begin{abstract}
We study the problems of finding the conditions for the existence of solutions of a linear Noetherian boundary-value problem for a hybrid system of ordinary differential equations and difference equations and the construction of these solutions. We also propose an algorithm for the construction of solutions of linear Noetherian boundary-value problems for hybrid systems of difference-differential equations in the critical and noncritical cases.
\end{abstract}

2010 Mathematics Subject Classification: 34B10; 34B15

Keywords: boundary value problems, matrix differential equations

\section{INTRODUCTION}

The mathematical description of continuous processes with short-term perturbations whose duration can be neglected leads to the investigation of boundary-value problems for impulsive ordinary differential equations [7, 13, 14, 19, 22, 24, 25]. The analysis of discrete processes requires the study of boundary-value problems for difference equations $[3,5,20,21,23]$. The proposed hybrid difference-differential system contains an unknown function of piecewise continuous argument and an unknown function of discrete argument. To find these unknowns, we propose to use a system of ordinary differential equations containing the unknown function of discrete argument and a system of difference equations containing the unknown function of piecewise continuous argument. The proposed approach to the mathematical description of both continuous and discrete processes enables one to combine the efficient methods of the theory of boundary-value problems for ordinary differential and difference equations.

\section{STATEMENT OF THE PROBLEM}

We consider the problem of finding conditions for the existence of solutions

$$
\begin{gathered}
x(t) \in \mathbb{R}^{n}, t \in \Omega \cup \Theta, \quad \Omega:=\left[a, \tau_{1}\left[\cup \left[\tau_{p_{1}}, \tau_{p_{1}+1}\left[\cup \ldots \cup\left[\tau_{p_{q}}, \tau_{p_{q}+1}\right] \subset[a, b],\right.\right.\right.\right. \\
y(k) \in \mathbb{R}^{m}, \quad k \in \Theta:=\left\{a, \tau_{1}, \tau_{2}, \ldots, \tau_{p_{1}}, \tau_{p_{1}+1}, \ldots, \tau_{p_{2}}, \tau_{p_{2}+1}, \ldots, b\right\}
\end{gathered}
$$


and the problem of construction of these solutions for the hybrid Noetherian $(m+n \neq$ $\lambda+\mu$ ) difference-differential boundary-value problem

$$
\begin{gathered}
x^{\prime}(t)=A(t, k) x(t)+B(t, k) y(k)+\varphi(t, k), \quad t \in \Omega, \\
y(k+1)=C(k) x(k)+D(k) y(k)+\psi(k), \quad k \in \Theta, \\
\Delta x\left(\tau_{p_{i}+1}, \tau_{p_{i+1}}\right):=S_{\tau_{p_{i}+1}} x\left(\tau_{p_{i}+1}-0\right)+b_{\tau_{p_{i}+1}}, \quad \ell x(\cdot)=\alpha, \quad \wp y(\cdot)=\beta .
\end{gathered}
$$

We seek the first component of the required solution $x(t) \in \mathbb{C}^{1}\left[\Omega_{I}\right]$ of the boundaryvalue problem (2.1)-(2.3) in a class of functions continuously differentiable on the set $\Omega \subset[a, b]$, except the points $\tau_{1}, \tau_{p_{1}+1}, \tau_{p_{2}+1}, \ldots ., \tau_{p_{q-1}}$ at which the solution $x(t)$ may have finite discontinuities

$$
\Delta x\left(\tau_{p_{i}+1}, \tau_{p_{i+1}}\right):=x\left(\tau_{p_{i+1}}\right)-x\left(\tau_{p_{i}+1}-0\right) \leq \rho, \quad i=0,1,2, \ldots, q-1,
$$

where $a:=\tau_{0}:=\tau_{p_{0}}, \tau_{p_{q}+1}:=b, \alpha \in \mathbb{R}^{\mu}, \beta \in \mathbb{R}^{v}$. The second component of the required solution $y(k) \in \mathbb{R}^{m}, k \in \Theta$ of the Noetherian boundary-value problem (2.1)-(2.3) is sought in the set of bounded sequences. The matrices $A(t, k) \in \mathbb{R}^{n \times n}$, $B(t, k) \in \mathbb{R}^{n \times m}$ and the vector function $\varphi(t, k) \in \mathbb{R}^{n}$ are assumed to be continuous in the first and second arguments on the segment $[a, b]$. The matrices $C(k) \in$ $\mathbb{R}^{m \times n}, D(k) \in \mathbb{R}^{m \times m}, S_{\tau_{i}} \in \mathbb{R}^{m \times m}$ and the vector function $\psi(k) \in \mathbb{R}^{m}$ are assumed to be bounded on the set $\Theta$ by functions, $\ell x(\cdot)$ is the linear bounded vector functional:

$$
\ell x(\cdot): \mathbb{C}^{1}\left[\Omega_{I}\right] \rightarrow \mathbb{R}^{\mu},
$$

$\wp y(\cdot)$ is the linear bounded vector functional given in the space of vector functions bounded on the set $\Theta$ as follows:

$$
\wp y(\cdot):\left\{y(k): \Theta \rightarrow \mathbb{R}^{m}\right\} \rightarrow \mathbb{R}^{v}, \quad \mu \neq v .
$$

Various hybrid boundary-value problems for systems of ordinary differential and functional-differential equations are extensively investigated (see, e.g., [3]). The posed hybrid boundary-value problem (2.1)-(2.3) is a generalization of boundaryvalue problems for ordinary impulsive differential equations [7, 13, 19, 22, 24, 25], boundary-value problems for difference equations [5,20,21,23], and different boundaryvalue problems for systems of ordinary differential equations [7].

\section{Generalized GREen OPERATOR OF THE CAUCHY PROBLEM}

For the sake of definiteness, we set

$$
x(a)=\zeta \in \mathbb{R}^{n}, \quad y(a)=\xi \in \mathbb{R}^{m}, \quad m \neq n .
$$

Let $X_{a}(t)$ be the normal $\left(X_{a}(a)=I_{n}\right)$ fundamental matrix of the homogeneous part $x^{\prime}(t)=A(t, a) x(t)$ of system (2.1) on the segment $\left[a ; \tau_{1}\right]$. The solution of system (2.1) in the interval $\left[a ; \tau_{1}\right.$ [ is represented in the form

$$
x(t, \zeta, \xi)=X_{a}(t) \zeta+V_{a}(t) \xi+K_{a}[\varphi(s, a)](t)
$$




$$
y(a, \zeta, \xi)=Z(a) \zeta+W(a) \xi+K\left[\psi\left(\tau_{0}\right)\right](a), Z\left(\tau_{0}\right):=0, W\left(\tau_{0}\right):=I_{m},
$$

where

$$
V_{a}(t):=X_{a}(t) \int_{a}^{t} X_{a}^{-1}(s) B(s, a) W(a) d s, K\left[\psi\left(\tau_{0}\right)\right](a)=0
$$

and

$$
K_{a}[\varphi(s, a)](t):=X_{a}(t) \int_{a}^{t} X_{a}^{-1}(s) \varphi(s, a) d s
$$

is the generalized Green operator of the Cauchy problem (3.1) for system (2.1) in the interval $\left[a ; \tau_{1}\left[\right.\right.$. We represent the solution of system (2.2) for $k=\tau_{1}$ in the form

$y\left(\tau_{1}, \zeta, \xi\right)=Z\left(\tau_{1}\right) \zeta+W\left(\tau_{1}\right) \xi+K[\psi(a)]\left(\tau_{1}\right), Z\left(\tau_{1}\right):=C\left(\tau_{0}\right), \quad W\left(\tau_{1}\right):=D\left(\tau_{0}\right)$,

where

$$
K[\psi(a)]\left(\tau_{1}\right)=\psi\left(\tau_{0}\right)=\psi(a)
$$

is the generalized Green operator of the Cauchy problem $y(a)=\xi$ for system (2.2) with $k=\tau_{1}$. Similarly, we represent the solution of system (2.2) for $k=\tau_{2}$ in the form

where

$$
y\left(\tau_{2}, \zeta, \xi\right)=Z\left(\tau_{2}\right) \zeta+W\left(\tau_{2}\right) \xi+K\left[\psi\left(\tau_{1}\right)\right]\left(\tau_{2}\right)
$$

$$
\begin{gathered}
Z\left(\tau_{2}\right):=C\left(\tau_{1}\right) X_{a}\left(\tau_{1}\right)+D\left(\tau_{1}\right) Z\left(\tau_{1}\right), \quad W\left(\tau_{2}\right):=C\left(\tau_{1}\right) V_{a}\left(\tau_{1}\right)+D\left(\tau_{1}\right) W\left(\tau_{1}\right), \\
K\left[\psi\left(\tau_{1}\right)\right]\left(\tau_{2}\right):=C\left(\tau_{1}\right) K_{a}[\varphi(s, a)]\left(\tau_{1}\right)+D\left(\tau_{1}\right) K[\psi(a)]\left(\tau_{1}\right)+\psi\left(\tau_{1}\right) .
\end{gathered}
$$

If we continue our reasoning, then we get a solution of system (2.2) with $k=\tau_{p_{1}}$ :

$$
y\left(\tau_{p_{1}}, \zeta, \xi\right)=Z\left(\tau_{p_{1}}\right) \zeta+W\left(\tau_{p_{1}}\right) \xi+K\left[\psi\left(\tau_{p_{1}}-1\right)\right]\left(\tau_{p_{1}}\right)
$$

where

$$
\begin{aligned}
Z\left(\tau_{p_{1}}\right) & :=C\left(\tau_{p_{1}-1}\right) X_{a}\left(\tau_{p_{1}-1}\right)+D\left(\tau_{p_{1}-1}\right) Z\left(\tau_{p_{1}-1}\right), \\
W\left(\tau_{p_{1}}\right) & :=C\left(\tau_{p_{1}-1}\right) V_{a}\left(\tau_{p_{1}-1}\right)+D\left(\tau_{p_{1}-1}\right) W\left(\tau_{p_{1}-1}\right)
\end{aligned}
$$

and

$$
\begin{aligned}
K\left[\psi\left(\tau_{p_{1}-1}\right)\right]\left(\tau_{p_{1}}\right):= & C\left(\tau_{p_{1}-1}\right) K_{a}\left[\varphi\left(s, \tau_{0}\right)\right]\left(\tau_{p_{1}-1}\right) \\
& +D\left(\tau_{p_{1}-1}\right) K\left[\psi\left(\tau_{p_{1}-2}\right)\right]\left(\tau_{p_{1}-1}\right)+\psi\left(\tau_{p_{1}-1}\right)
\end{aligned}
$$


is the generalized Green operator of the Cauchy problem $y(a)=\xi$ for system (2.2) with $k=\tau_{p_{1}}$. Let $X_{1}(t)$ be the normal $\left(X_{1}\left(\tau_{p_{1}}\right)=I_{n}\right)$ fundamental matrix of the homogeneous part

$$
x^{\prime}(t)=A\left(t, \tau_{p_{1}}\right) x(t), \quad t \in\left[\tau_{p_{1}} ; \tau_{p_{1}+1}[\right.
$$

of system (2.1). We represent the solution of system (2.1) satisfying the first condition in (2.3) on the interval $\left[\tau_{p_{1}} ; \tau_{p_{1}+1}[\right.$ in the form

$$
x(t, \zeta, \xi)=X_{\tau_{p_{1}}}(t) \zeta+V_{\tau_{p_{1}}}(t) \xi+K_{\tau_{p_{1}}}\left[\varphi\left(s, \tau_{p_{1}}\right) ; b_{1}\right](t)
$$

where

$$
K_{\tau_{p_{1}}}\left[\varphi\left(s, \tau_{p_{1}}\right)\right](t):=X_{1}(t) \int_{\tau_{p_{1}}}^{t} X_{1}^{-1}(s) \varphi\left(s, \tau_{p_{1}}\right) d s, \quad t \in\left[\tau_{p_{1}} ; \tau_{p_{1}+1}[\right.
$$

is the Green operator of the Cauchy problem (3.1) for system (2.1),

$$
X_{\tau_{p_{1}}}(t):=X_{1}(t)\left(I_{n}+S_{1}\right) X_{a}\left(\tau_{1}\right)+K_{\tau_{p_{1}}}\left[B\left(s, \tau_{p_{1}}\right) Z\left(\tau_{p_{1}}\right)\right](t)
$$

is the normal $\left(X_{\tau_{p_{1}}}\left(\tau_{p_{1}}\right)=\left(I_{n}+S_{1}\right) X_{a}\left(\tau_{1}\right)\right)$ fundamental matrix of the homogeneous part of system (2.1) for $t \in\left[\tau_{p_{1}} ; \tau_{p_{1}+1}\right.$ [ satisfying the first condition in (2.3)

$$
\begin{aligned}
& V_{\tau_{p_{1}}}(t):=X_{1}(t)\left(I_{n}+S_{1}\right) V_{a}\left(\tau_{1}\right)+K_{\tau_{p_{1}}}\left[B\left(s, \tau_{p_{1}}\right) W\left(\tau_{p_{1}}\right)\right](t), \\
& \mathcal{K}_{\tau_{p_{1}}}\left[\varphi\left(s, \tau_{p_{1}}\right) ; b_{1}\right](t):=X_{1}(t) b_{1}+X_{1}(t)\left(I_{n}+S_{1}\right) K_{a}[\varphi(s, a)]\left(\tau_{1}\right) \\
&+K_{\tau_{p_{1}}}\left[\varphi\left(s, \tau_{p_{1}}\right)\right](t)+K_{\tau_{p_{1}}}\left\{B\left(s, \tau_{p_{1}}\right) K\left[\psi\left(\tau_{p_{1}-1}\right)\right]\left(\tau_{p_{1}}\right)\right\}(t) .
\end{aligned}
$$

Note that the function $x(t, \zeta, \xi)$ is defined both on the segment $\left[\tau_{p_{1}} ; \tau_{p_{1}+1}\right]$ and at the points

$$
t=k \in\left\{\tau_{p_{1}}, \tau_{p_{1}+1}, \ldots, \tau_{p_{2}-1}\right\} \in \Theta .
$$

We represent the solution of system (2.2) for $k=\tau_{p_{1}+1}$ in the form

$$
y\left(\tau_{p_{1}+1}, \zeta, \xi\right)=Z\left(\tau_{p_{1}+1}\right) \zeta+W\left(\tau_{p_{1}+1}\right) \xi+\mathcal{K}\left[\psi\left(\tau_{p_{1}}\right) ; b_{1}\right]\left(\tau_{p_{1}+1}\right)
$$

where

$$
\begin{aligned}
Z\left(\tau_{p_{1}+1}\right) & :=C\left(\tau_{p_{1}}\right) X_{\tau_{p_{1}}}\left(\tau_{p_{1}}\right)+D\left(\tau_{p_{1}}\right) Z\left(\tau_{p_{1}}\right), \\
W\left(\tau_{p_{1}+1}\right) & :=C\left(\tau_{p_{1}}\right) V_{\tau_{p_{1}}}\left(\tau_{p_{1}}\right)+D\left(\tau_{p_{1}}\right) W\left(\tau_{p_{1}}\right)
\end{aligned}
$$

and

$$
\mathcal{K}\left[\psi\left(\tau_{p_{1}}\right) ; b_{1}\right]\left(\tau_{p_{1}+1}\right):=C\left(\tau_{p_{1}}\right) \mathcal{K}_{\tau_{p_{1}}}\left[\varphi\left(s, \tau_{p_{1}}\right) ; b_{1}\right]\left(\tau_{p_{1}}\right)
$$




$$
+D\left(\tau_{p_{1}}\right) K\left[\psi\left(\tau_{p_{1}-1}\right)\right]\left(\tau_{p_{1}}\right)+\psi\left(\tau_{p_{1}}\right)
$$

is the generalized Green operator of the Cauchy problem $y(a)=\xi$ for system (2.2). Continuing our reasoning, we determine a solution of system (2.2) with $k=\tau_{p_{2}}$ :

$$
y\left(\tau_{p_{2}}, \zeta, \xi\right)=Z\left(\tau_{p_{2}}\right) \zeta+W\left(\tau_{p_{2}}\right) \xi+\mathcal{K}\left[\psi\left(\tau_{p_{2}}-1\right) ; b_{1}\right]\left(\tau_{p_{2}}\right),
$$

where

$$
\begin{aligned}
Z\left(\tau_{p_{2}}\right) & :=C\left(\tau_{p_{2}-1}\right) X_{\tau_{p_{1}}}\left(\tau_{p_{2}-1}\right)+D\left(\tau_{p_{2}-1}\right) Z\left(\tau_{p_{2}-1}\right), \\
W\left(\tau_{p_{2}}\right) & :=C\left(\tau_{p_{2}-1}\right) V_{\tau_{p_{1}}}\left(\tau_{p_{2}-1}\right)+D\left(\tau_{p_{2}-1}\right) W\left(\tau_{p_{2}-1}\right)
\end{aligned}
$$

and

$$
\begin{aligned}
\mathcal{K}\left[\psi\left(\tau_{p_{2}}-1\right) ; b_{1}\right]\left(\tau_{p_{2}}\right):= & C\left(\tau_{p_{2}-1}\right) \mathcal{K}_{\tau_{p_{1}}}\left[\varphi\left(s, \tau_{p_{2}-2}\right) ; b_{1}\right]\left(\tau_{p_{2}-1}\right) \\
& +D\left(\tau_{p_{2}-1}\right) K\left[\psi\left(\tau_{p_{2}-2}\right)\right]\left(\tau_{p_{2}-1}\right)+\psi\left(\tau_{p_{2}-1}\right)
\end{aligned}
$$

is the generalized Green operator of the Cauchy problem $y(a)=\xi$ for system (2.2). Further, we continue our reasoning and assume that $X_{q}(t)$ is the normal $\left(X_{q}\left(\tau_{p_{q}}\right)=\right.$ $I_{n}$ ) fundamental matrix of the homogeneous part

$$
x^{\prime}(t)=A\left(t, \tau_{p_{q}}\right) x(t), \quad t \in\left[\tau_{p_{q}} ; b\right],
$$

of system (2.1). We represent the solution of system (2.1) satisfying the first condition in $(2.3)$ on the segment $\left[\tau_{p_{q}} ; b\right]$ in the form

$$
x(t, \zeta, \xi)=X_{\tau_{p_{q}}}(t) \zeta+V_{\tau_{p_{q}}}(t) \xi+\mathcal{K}_{\tau_{p_{q}}}\left[\varphi\left(s, \tau_{p_{1}}\right) ; b_{q}\right](t),
$$

where

$$
K_{\tau_{p_{q}}}\left[\varphi\left(s, \tau_{p_{q}}\right)\right](t):=X_{q}(t) \int_{\tau_{p_{q}}}^{t} X_{q}^{-1}(s) \varphi\left(s, \tau_{p_{1}}\right) d s, \quad t \in\left[\tau_{p_{q}} ; b\right],
$$

is the Green operator of the Cauchy problem (3.1) for system (2.1),

$$
X_{\tau_{p_{q}}}(t):=X_{q}(t)\left(I_{n}+S_{q}\right) X_{p_{q-1}}\left(\tau_{q}\right)+K_{\tau_{p_{q}}}\left[B\left(s, \tau_{p_{q}}\right) Z\left(\tau_{p_{q}}\right)\right](t)
$$

is the normal $\left(X_{\tau_{p_{q}}}\left(\tau_{p_{q}}\right)=\left(I_{n}+S_{q}\right) X_{p_{q-1}}\left(\tau_{q}\right)\right)$ fundamental matrix of the homogeneous part of system (2.1) for $t \in\left[\tau_{p_{q}} ; b\right]$ satisfying the first condition in (2.3),

$$
\begin{aligned}
V_{\tau_{p_{q}}}(t) & :=X_{q}(t)\left(I_{n}+S_{q}\right) V_{\tau_{p_{q-1}}}\left(\tau_{p_{q}}\right)+\mathcal{K}_{\tau_{p_{q}}}\left[B\left(s, \tau_{p_{q}}\right) W\left(\tau_{p_{q}}\right)\right](t), \\
\mathcal{K}_{\tau_{p_{q}}}\left[\varphi\left(s, \tau_{p_{q}}\right) ; b_{q}\right](t) & :=X_{q}(t) b_{q}+X_{q}(t)\left(I_{n}+S_{q}\right) \mathcal{K}_{\tau_{p_{q}}}\left[\varphi\left(s, \tau_{p_{q-1}}\right) ; b_{q-1}\right]\left(\tau_{p_{q}}\right)
\end{aligned}
$$




$$
+K_{\tau_{p q}}\left[\varphi\left(s, \tau_{p_{q}}\right)\right](t)+K_{\tau_{p_{q}}}\left\{B\left(s, \tau_{p_{q}}\right) \mathcal{K}\left[\psi\left(\tau_{p_{1}-1}\right) ; b_{q-1}\right]\left(\tau_{p_{q}}\right)\right\}(t) .
$$

Lemma 1. The general solution of the Cauchy problem (3.1) for system (2.1), (2.2) satisfying the first condition in (2.3) can be represented in the form

$$
\begin{gathered}
x(t, \zeta, \xi)=X_{\tau_{p_{i}}}(t) \zeta+V_{\tau_{p_{i}}}(t) \xi+\mathcal{K}_{\tau_{p_{i}}}\left[\varphi\left(s, \tau_{i}\right)\right](t), \\
y(k, \zeta, \xi)=Z(k) \zeta+W(k) \xi+\mathcal{K}\left[\psi\left(k-1, \tau_{i}\right)\right](k),
\end{gathered}
$$

where the matrices

$$
\begin{aligned}
V_{a}(t) & :=X_{a}(t) \int_{a}^{t} X_{a}^{-1}(s) B(s, a) W(a) d s, \quad t \in\left[a ; \tau_{1}[,\right. \\
Z\left(\tau_{p_{1}}\right) & :=C\left(\tau_{p_{1}-1}\right) X_{a}\left(\tau_{p_{1}-1}\right)+D\left(\tau_{p_{1}-1}\right) Z\left(\tau_{p_{1}-1}\right), \quad Z(a):=0, \\
W\left(\tau_{p_{1}}\right) & :=C\left(\tau_{p_{1}-1}\right) V_{a}\left(\tau_{p_{1}-1}\right)+D\left(\tau_{p_{1}-1}\right) W\left(\tau_{p_{1}-1}\right), W(a):=I_{m}, \ldots, \\
X_{\tau_{p_{q}}}(t) & =X_{q}(t)\left(I_{n}+S_{q}\right) X_{p_{q-1}}\left(\tau_{q}\right)+\mathcal{K}_{\tau_{p_{q}}}\left[B\left(s, \tau_{p_{q}}\right) Z\left(\tau_{p_{q}}\right)\right](t), \quad t \in\left[\tau_{p_{q}} ; b\right], \\
V_{\tau_{p_{q}}}(t) & =X_{q}(t)\left(I_{n}+S_{q}\right) V_{\tau_{p_{q-1}}}\left(\tau_{p_{q}}\right)+\mathcal{K}_{\tau_{p_{q}}}\left[B\left(s, \tau_{p_{q}}\right) W\left(\tau_{p_{q}}\right)\right](t), \quad t \in\left[\tau_{p_{q}} ; b\right],
\end{aligned}
$$

specify the general solution

$$
x(t, \zeta, \xi)=X_{\tau_{p_{i}}}(t) \zeta+V_{\tau_{p_{i}}}(t) \xi, \quad y(k, \zeta, \xi)=Z(k) \zeta+W(k) \xi
$$

of the homogeneous part of system (2.1), (2.2) satisfying the first condition in (2.3) and

$$
\begin{aligned}
& \mathcal{K}_{a}[\varphi(s, a)](t):=X_{a}(t) \int_{a}^{t} X_{a}^{-1}(s) \varphi(s, a) d s, t \in\left[a ; \tau_{1}\right], \\
& K[\psi(a)]\left(\tau_{1}\right):=\psi\left(\tau_{1}\right), \\
& K\left[\psi\left(\tau_{1}\right)\right]\left(\tau_{2}\right):=C\left(\tau_{1}\right) K_{a}[\varphi(s, a)]\left(\tau_{1}\right)+D\left(\tau_{1}\right) K[\psi(a)]\left(\tau_{1}\right)+\psi\left(\tau_{1}\right), \ldots, \\
& K\left[\psi\left(\tau_{p_{1}-1}\right)\right]\left(\tau_{p_{1}}\right):=C\left(\tau_{p_{1}-1}\right) K_{a}\left[\varphi\left(s, \tau_{0}\right)\right]\left(\tau_{p_{1}-1}\right) \\
& \quad+D\left(\tau_{p_{1}-1}\right) K\left[\psi\left(\tau_{p_{1}-2}\right)\right]\left(\tau_{p_{1}-1}\right)+\psi\left(\tau_{p_{1}-1}\right), \mathcal{K}\left[\psi\left(\tau_{p_{1}}\right) ; b_{1}\right]\left(\tau_{p_{1}+1}\right) \\
& \quad:=C\left(\tau_{p_{1}}\right) \mathcal{K}_{\tau_{p_{1}}}\left[\varphi\left(s, \tau_{p_{1}}\right) ; b_{1}\right]\left(\tau_{p_{1}}\right)+D\left(\tau_{p_{1}}\right) K\left[\psi\left(\tau_{p_{1}-1}\right)\right]\left(\tau_{p_{1}}\right)+\psi\left(\tau_{p_{1}}\right), \ldots, \\
& \mathcal{K}_{\tau_{p_{q}}}\left[\varphi\left(s, \tau_{p_{q}}\right) ; b_{q}\right](t)=X_{q}(t) b_{q}+X_{q}(t)\left(I_{n}+S_{q}\right) \mathcal{K}_{\tau_{p_{q}}}\left[\varphi\left(s, \tau_{p_{q-1}}\right) ; b_{q-1}\right]\left(\tau_{p_{q}}\right)
\end{aligned}
$$




$$
\begin{aligned}
& +K_{\tau_{p_{q}}}\left[\varphi\left(s, \tau_{p_{q}}\right)\right](t)+K_{\tau_{p_{q}}}\left\{B\left(s, \tau_{p_{q}}\right) \mathcal{K}\left[\psi\left(\tau_{p_{1}-1}\right) ; b_{q-1}\right]\left(\tau_{p_{q}}\right)\right\}(t), \quad t \in\left[\tau_{p_{q}} ; b\right], \\
& \mathcal{K}\left[\psi\left(\tau_{p_{q}}-1\right) ; b_{q}\right]\left(\tau_{p_{q}}\right):=C\left(\tau_{p_{q}-1}\right) \mathcal{K}_{\tau_{p_{q}}}\left[\varphi\left(s, \tau_{p_{q}-2}\right) ; b_{q}\right]\left(\tau_{p_{q}-1}\right) \\
& +D\left(\tau_{p_{q}-1}\right) K\left[\psi\left(\tau_{p_{q}-2}\right)\right]\left(\tau_{p_{q}-1}\right)+\psi\left(\tau_{p_{q}-1}\right)
\end{aligned}
$$

is the generalized Green operator of the Cauchy problem (3.1) for system (2.1), (2.2) satisfying the first condition in (2.3).

\section{Generalized GREen OPERATOR OF THE HYBRID DIFFERENCE-DIFFERENTIAL BOUNDARY-VALUE PROBLEM}

In what follows, we study the problem of determination of conditions for the existence of solutions of the linear boundary-value problem (2.1)-(2.3) and construct these solutions. Assume that the conditions of the lemma are satisfied. We represent the linear bounded vector functional $\ell x(\cdot)$ in the form

$$
\ell x(\cdot):=\sum_{i=0}^{q} \ell_{i} x(\cdot)
$$

where

$$
\ell_{i} x(\cdot): \mathbb{C}^{1}\left[\tau_{p_{i}}, \tau_{p_{i}+1}\right] \rightarrow \mathbb{R}^{m}, \quad i=0,1,2, \ldots, q
$$

are linear bounded vector functionals. Further, we enumerate elements of the set $\Theta$ :

$$
k_{i} \in \Theta: k_{0}:=a, k_{1}:=\tau_{1}, \ldots, k_{\sigma}:=b, \quad \sigma:=p_{1}+\ldots p_{q}+2 .
$$

Similarly, we represent the linear bounded vector functional $\wp y(\cdot)$ in the form

$$
\wp y(\cdot):=\sum_{k \in \Theta} \wp_{k} y(\cdot),
$$

where $\wp_{k} y(\cdot):\left\{y(k): \Theta \rightarrow \mathbb{R}^{m}\right\} \rightarrow \mathbb{R}^{v}$ are linear bounded vector functionals. Denote the vectors

$$
\lambda_{\ell}:=\sum_{i=0}^{q} \ell_{i} \mathcal{K}_{\tau_{p_{i}}}\left[\varphi\left(s, \tau_{i}\right)\right](\cdot) \in \mathbb{R}^{\mu}
$$

and

$$
\lambda_{\wp}:=\sum_{i=0}^{\sigma} \wp_{i} \mathcal{K}\left[\psi\left(k_{i}\right) ; b_{j}\right]\left(k_{i}+1\right), \quad j=0,1,2, \ldots, q .
$$

Substituting the solution of the Cauchy problem (3.1) for system (2.1), (2.2) satisfying the first condition in (2.3) in the second and third conditions in (2.3), we arrive 
at the problem of determination of solutions $\zeta \in \mathbb{R}^{n}, \xi \in \mathbb{R}^{m}$ of the linear matrix equation

$$
\mathcal{Q}\left(\begin{array}{l}
\zeta \\
\xi
\end{array}\right)=\lambda, \quad \lambda:=\left(\begin{array}{c}
\alpha-\lambda_{\ell} \\
\beta-\lambda_{\wp}
\end{array}\right) .
$$

In the critical case $\left(P_{Q^{*}} \neq 0\right)$, under the condition

$$
P_{Q_{d}^{*}} \lambda=0,
$$

the solution of the last equation is given by the vector

$$
\left(\begin{array}{l}
\zeta \\
\xi
\end{array}\right)=Q^{+} \lambda+P_{\mathcal{Q}_{r}} c_{r}, \quad c_{r} \in \mathbb{R}^{r},
$$

where

$$
\mathcal{Q}:=\left(\begin{array}{cc}
\ell X(\cdot) & \ell V(\cdot) \\
\wp Z(\cdot) & \wp W(\cdot)
\end{array}\right)
$$

is an $((\mu+v) \times(m+n))$ constant matrix. Here, $P_{Q^{*}}$ is the $((\mu+v) \times(\mu+v))$ matrix orthoprojector $P_{Q^{*}}: \mathbb{R}^{\mu+v} \rightarrow \mathbb{N}\left(Q^{*}\right)$; the matrix $P_{Q_{r}}$ is formed by $r$ linearly independent columns of the $((m+n) \times(m+n))$ matrix orthoprojector $P_{\mathcal{Q}}$, and the matrix $P_{Q_{d}^{*}}$ is formed by $d$ linearly independent rows of the matrix orthoprojector $P_{Q^{*} \text {. In addition, }}$

$$
\begin{gathered}
\ell X(\cdot):=\sum_{i=0}^{q} \ell_{i} X_{\tau_{p_{i}}}(\cdot), \quad \ell V(\cdot):=\sum_{i=0}^{q} \ell_{i} V_{\tau_{p_{i}}}(\cdot), \\
\wp Z(\cdot):=\sum_{i=0}^{\sigma} \wp_{i} Z\left(k_{i}\right), \quad \wp W(\cdot):=\sum_{i=0}^{\sigma} \wp_{i} W\left(k_{i}\right) .
\end{gathered}
$$

In the critical case, under condition (4.1), the solution of the boundary-value problem (2.1)-(2.3) specifies an $r$-parameter family of vectors $\zeta \in \mathbb{R}^{n}, \xi \in \mathbb{R}^{m}$. We denote the blocks of the matrix orthoprojector $P_{\mathcal{Q}}$ as follows:

$$
\begin{aligned}
& P_{\mathcal{Q}_{r_{(11)}}}:=\left(\begin{array}{cc}
I_{n} & O
\end{array}\right) P_{\mathcal{Q}_{r}}, \quad P_{\mathcal{Q}_{r_{(12)}}}:=\left(\begin{array}{ll}
O & I_{n}
\end{array}\right) P_{\mathcal{Q}_{r}}, \\
& P_{Q_{r_{(21)}}}:=\left(\begin{array}{cc}
I_{m} & O
\end{array}\right) P_{Q_{r}}, \quad P_{Q_{r_{(22)}}}:=\left(\begin{array}{cc}
O & I_{m}
\end{array}\right) P_{Q_{r}} .
\end{aligned}
$$

For the matrices, we write

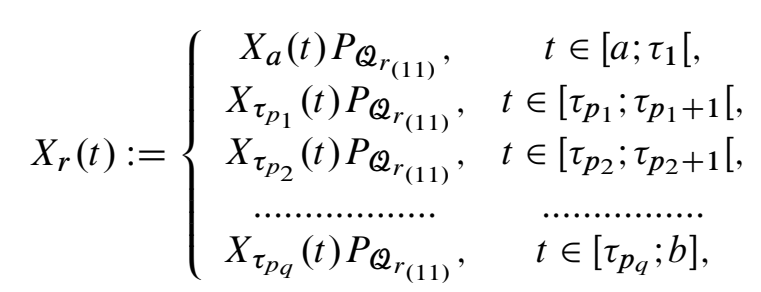




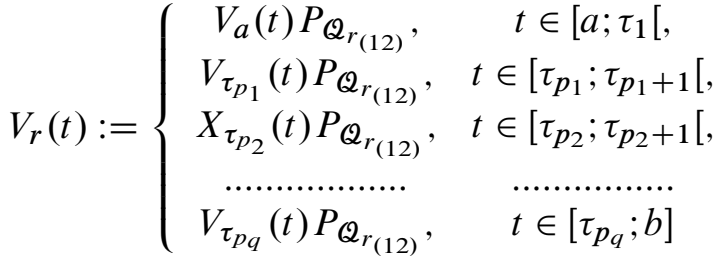

$$
\begin{aligned}
& Z_{r}(k):=Z(k) P_{\mathcal{Q}_{r_{(21)}}}, \quad W_{r}(k):=W(k) P_{\mathcal{Q}_{r_{(22)}}}, \quad k \in \Theta .
\end{aligned}
$$

We also denote the blocks of the matrix $\mathcal{Q}^{+}$as

$$
\begin{aligned}
& \mathcal{Q}_{(11)}^{+}:=\left(\begin{array}{cc}
I_{n} & O
\end{array}\right) \mathbb{Q}^{+}, \quad Q_{(12)}^{+}:=\left(\begin{array}{ll}
O & I_{n}
\end{array}\right) \mathcal{Q}^{+}, \\
& \mathcal{Q}_{(21)}^{+}:=:=\left(\begin{array}{ll}
I_{m} & O
\end{array}\right) \mathcal{Q}^{+}, Q_{(22)}^{+}:=:=\left(\begin{array}{cc}
O & I_{m}
\end{array}\right) \mathcal{Q}^{+} \text {. }
\end{aligned}
$$

The theorem presented below gives necessary and sufficient conditions for the solvability of the hybrid difference-differential boundary-value problem (2.1)-(2.3).

Theorem 1. In the critical case $\left(P_{Q^{*}} \neq 0\right)$, under condition (4.1), the solution of the hybrid difference-differential boundary-value problem (2.1)-(2.3) can be represented in the form

$$
\begin{aligned}
& x\left(t, c_{r}\right)=X_{r}(t) c_{r}+V_{r}(t) c_{r}+G\left[\varphi\left(s, \tau_{i}\right) ; \alpha\right](t), \quad c_{r} \in \mathbb{R}^{r}, \\
& y\left(k, c_{r}\right)=Z_{r}(k) c_{r}+W_{r}(k) c_{r}+G\left[\psi\left(k-1, \tau_{i}\right) ; \beta\right](k)
\end{aligned}
$$

with the help of the generalized Green operator of the linear boundary-value problem (2.1)-(2.3)

$$
\begin{aligned}
& G\left[\varphi\left(s, \tau_{i}\right) ; \alpha\right](t):= \\
& =\left\{\begin{array}{cc}
X_{a}(t) \mathcal{Q}_{(11)}^{+} \lambda+V_{a}(t) \mathcal{Q}_{(12)}^{+} \mu+\mathcal{K}_{a}[\varphi(s, a)](t), & t \in\left[a ; \tau_{1}[,\right. \\
X_{\tau_{p_{1}}}(t) \mathcal{Q}_{(11)}^{+} \lambda+V_{\tau_{p_{1}}}(t) \mathcal{Q}_{(12)}^{+} \mu+\mathcal{K}_{\tau_{p_{1}}}\left[\varphi\left(s, \tau_{p_{1}} ; b_{1}\right)\right](t), & t \in\left[\tau_{p_{1}} ; \tau_{p_{1}+1}[,\right. \\
\ldots \ldots \ldots \ldots \ldots . . . \ldots \ldots \ldots \ldots \ldots \ldots \ldots \\
X_{\tau_{p_{q}}}(t) \mathcal{Q}_{(11)}^{+} \lambda+V_{\tau_{p_{q}}}(t) \mathcal{Q}_{(12)}^{+} \mu+\mathcal{K}_{\tau_{p_{q}}}\left[\varphi\left(s, \tau_{p_{q}} ; b_{q}\right)\right](t), & t \in\left[\tau_{p_{q}} ; b\right],
\end{array}\right. \\
& G\left[\psi\left(k-1, \tau_{i}\right) ; \beta\right](k):=Z(k) \mathcal{Q}_{(21)}^{+} \lambda+W(k) \mathcal{Q}_{(22)}^{+} \mu+K[\psi(k-1)](k), \quad k \in \Theta,
\end{aligned}
$$

where the matrices $X_{r}(t), V_{r}(t), Z_{r}(k)$, and $W_{r}(k)$ specify the general solution of the homogeneous part of the boundary-value problem (2.1)-(2.3); here, $K[\varphi(s, k)](t)$, $K[\psi(k-1)](k)$ is the generalized Green operator of the Cauchy problem (3.1) for system (2.1), (2.2) satisfying the first condition in (2.3). 
The condition of solvability (4.1) and the structure of the generalized Green operator of the hybrid difference-differential boundary-value problem (2.1)-(2.3) generalize the conventional results of the theory of Noetherian boundary-value problems for impulsive ordinary differential equations $[7,13,22,24]$ and the corresponding results of the theory of boundary-value problems for difference equations [5, 21,23].

Example 1. The problem of construction of solutions of the hybrid differencedifferential system

$$
x^{\prime}(t)=A x(t)+B y(k)+\varphi(t), y(k+1)=C x(k)+D y(k)+\psi(k),
$$

satisfying the antiperiodic boundary condition with pulsed action

$$
\Delta x\left(\tau_{1}, \tau_{3}\right):=S_{1} x\left(\tau_{1}-0\right)+b_{\tau_{1}}, \quad \ell x(\cdot)=\alpha \in \mathbb{R}^{2}, \wp y(\cdot)=\beta \in \mathbb{R}^{2},
$$

where $\tau_{1}:=\pi, \tau_{p_{1}}:=2 \pi$,

$$
\begin{gathered}
A:=\left(\begin{array}{cc}
0 & 1 \\
-1 & 0
\end{array}\right), \quad B:=\left(\begin{array}{cc}
1 & 0 \\
0 & 0
\end{array}\right), \quad C:=\left(\begin{array}{cc}
0 & 0 \\
1 & 0
\end{array}\right), \quad D:=\left(\begin{array}{ll}
0 & 0 \\
0 & 1
\end{array}\right), \\
\varphi(t):=\left(\begin{array}{c}
\sin 3 t \\
\cos 3 t
\end{array}\right), \quad \psi(k):=\left(\begin{array}{c}
\sin k \\
\cos k
\end{array}\right), \quad \Omega:=\left[0, \pi\left[\cup[2 \pi, 3 \pi], \quad \tau_{2}:=\frac{3 \pi}{2},\right.\right. \\
S_{1}:=-2\left(\begin{array}{ll}
1 & 0 \\
0 & 1
\end{array}\right), \quad b_{\tau_{1}}:=\left(\begin{array}{c}
1 \\
0
\end{array}\right), \quad \alpha:=\left(\begin{array}{c}
-1 \\
2
\end{array}\right), \quad \beta:=\left(\begin{array}{c}
0 \\
2
\end{array}\right), \\
\Theta:=\left\{0, \pi, \frac{3 \pi}{2}, 2 \pi, 3 \pi\right\}, \quad \operatorname{lx}(\cdot):=x(0)+x(3 \pi), \quad \wp y(\cdot):=y(0)+y(3 \pi)
\end{gathered}
$$

satisfies the conditions of the proved Theorem 1.

The matrices

$$
X_{0}(t)=\left(\begin{array}{cc}
\cos t & \sin t \\
-\sin t & \cos t
\end{array}\right), \quad V_{0}(t)=\left(\begin{array}{cc}
\sin t & 0 \\
\cos t-1 & 0
\end{array}\right), \quad Z(0)=W(0)=I_{2}
$$

specify the general solution

$$
x(t, \zeta, \xi)=X_{0}(t) \zeta+V_{0}(t) \xi, \quad y(0, \zeta, \xi)=Z(0) \zeta+W(0) \xi, \quad \zeta \in \mathbb{R}^{2}, \quad \xi \in \mathbb{R}^{2}
$$

of the homogeneous part of system (4.2) on the interval $[0 ; \pi[$. Similarly, the matrices

$$
\begin{gathered}
Z(\pi)=\left(\begin{array}{ll}
0 & 0 \\
1 & 0
\end{array}\right), W(\pi)=\left(\begin{array}{ll}
0 & 0 \\
0 & 1
\end{array}\right), \\
Z\left(\frac{3 \pi}{2}\right)=\left(\begin{array}{ll}
0 & 0 \\
0 & 0
\end{array}\right), W\left(\frac{3 \pi}{2}\right)=\left(\begin{array}{ll}
0 & 0 \\
0 & 1
\end{array}\right)
\end{gathered}
$$

specify the general solution

$$
y(\pi, \zeta, \xi)=Z(\pi) \zeta+W(\pi) \xi, \quad y\left(\frac{3 \pi}{2}, \zeta, \xi\right)=Z\left(\frac{3 \pi}{2}\right) \zeta+W\left(\frac{3 \pi}{2}\right) \xi
$$


of the homogeneous part of system (4.2) with $k \in\left\{\pi, \frac{3 \pi}{2}\right\}$. The matrices

$$
\begin{aligned}
& X_{2 \pi}(t):=X_{0}(t), V_{2 \pi}(t)=\left(\begin{array}{cc}
2 \sin t & 0 \\
2 \cos t & 0
\end{array}\right), \\
& Z(2 \pi)=\left(\begin{array}{cc}
0 & 0 \\
0 & -1
\end{array}\right), W(2 \pi)=\left(\begin{array}{cc}
0 & 0 \\
-1 & 1
\end{array}\right)
\end{aligned}
$$

specify the general solution

$x(t, \zeta, \xi)=X_{2 \pi}(t) \zeta+V_{2 \pi}(t) \xi, \quad y(2 \pi, \zeta, \xi)=Z(2 \pi) \zeta+W(2 \pi) \xi, \quad \zeta \in \mathbb{R}^{2}, \quad \xi \in \mathbb{R}^{2}$

of the homogeneous part of system $(4.2)$ on the segment $[2 \pi ; 3 \pi]$ satisfying the first boundary condition in (4.3). Similarly, the matrices

$$
Z(3 \pi)=\left(\begin{array}{cc}
0 & 0 \\
1 & -1
\end{array}\right), \quad W(3 \pi)=\left(\begin{array}{cc}
0 & 0 \\
-1 & 1
\end{array}\right)
$$

specify the general solution

$$
y(3 \pi, \zeta, \xi)=Z(3 \pi) \zeta+W(3 \pi) \xi, \quad \zeta \in \mathbb{R}^{2}, \quad \xi \in \mathbb{R}^{2}
$$

of the homogeneous part of system (4.2) with $k=3 \pi$. Since

$$
\mathcal{Q}=\left(\begin{array}{cccc}
0 & 0 & 0 & 0 \\
0 & 0 & -2 & 0 \\
1 & 0 & 1 & 0 \\
1 & 0 & -1 & 2
\end{array}\right),
$$

we have $P_{Q^{*}} \neq 0$. Therefore, the problem of construction of solutions of the hybrid difference-differential boundary-value problem (4.2), (4.3) corresponds to the critical case; moreover, the condition of its solvability (4.1) is satisfied. A solution of the hybrid difference-differential boundary-value problem (4.2), (4.3) can be represented in the form

$$
\begin{aligned}
& x\left(t, c_{r}\right)=X_{r}(t) c_{r}+V_{r}(t) c_{r}+G\left[\varphi\left(s, \tau_{i}\right) ; \alpha\right](t), \quad c_{r} \in \mathbb{R}^{1}, \\
& y\left(k, c_{r}\right)=Z_{r}(k) c_{r}+W_{r}(k) c_{r}+G\left[\psi\left(k-1, \tau_{i}\right) ; \beta\right](k)
\end{aligned}
$$

with the help of the generalized Green operator of the linear boundary-value problem (4.2), (4.3)

$$
\begin{aligned}
G\left[\varphi\left(s, \tau_{i}\right) ; \alpha\right](t) & =K_{\tau_{p_{i}}}\left[\varphi\left(s, \tau_{i}\right) ; b_{i}\right](t), \\
G\left[\psi\left(k-1, \tau_{i}\right) ; \beta\right](k) & =K\left[\psi\left(k-1, \tau_{i}\right) ; b_{i}\right](k) .
\end{aligned}
$$


Here,

$$
\begin{aligned}
& K_{\tau_{p_{i}}}\left[\varphi(s, k) ; b_{i}\right](t)=\left\{\begin{array}{c}
\frac{1}{2}\left(\begin{array}{c}
2 \sin t \sin 2 t \\
-\sin t+\sin 3 t
\end{array}\right), \quad t \in[0, \pi[, \\
\frac{1}{2}\left(\begin{array}{c}
3 \cos t-\cos 3 t-2 \sin t \\
2-2 \cos t-3 \sin t+\sin 3 t
\end{array}\right), \quad t \in[2 \pi, 3 \pi],
\end{array}\right. \\
& K[\psi](0)=\left(\begin{array}{l}
0 \\
0
\end{array}\right), \quad K[\psi(0)](\pi)=\left(\begin{array}{l}
0 \\
1
\end{array}\right), \quad K[\psi(\pi)]\left(\frac{3 \pi}{2}\right)=\left(\begin{array}{l}
0 \\
0
\end{array}\right), \\
& K\left[\psi\left(\frac{3 \pi}{2}\right)\right](2 \pi)=\left(\begin{array}{c}
-1 \\
0
\end{array}\right), \quad K[\psi(2 \pi)](3 \pi)=\left(\begin{array}{l}
0 \\
0
\end{array}\right)
\end{aligned}
$$

is the generalized Green operator of the Cauchy problem

$$
x(a)=\zeta \in \mathbb{R}^{2}, y(a)=\xi \in \mathbb{R}^{2}
$$

for system (4.2), (4.3). Since rank $P_{\mathcal{Q}}=1$, we get

$$
P_{Q_{r}}=\left(\begin{array}{llll}
0 & 1 & 0 & 0
\end{array}\right)^{*}
$$

and, hence,

$$
\begin{gathered}
X_{r}(t)=\left(\begin{array}{c}
\sin t \\
\cos t
\end{array}\right), V_{r}(t) \equiv 0, \quad t \in \Omega \\
Z_{r}(0)=-Z_{r}(3 \pi)=\left(\begin{array}{c}
0 \\
1
\end{array}\right), \quad W_{r}(0)=W_{r}(3 \pi)=\left(\begin{array}{l}
0 \\
0
\end{array}\right) .
\end{gathered}
$$

In the noncritical case $\left(P_{Q^{*}}=0\right)$, condition (4.1) is satisfied for any inhomogeneities $\varphi(t, k) \in \mathbb{R}^{n}, t \in \Omega$, and $\psi(k) \in \mathbb{R}^{m}, k \in \Theta$. Moreover, the solution of the hybrid difference-differential boundary-value problem (2.1)-(2.3) is determined by the following assertion:

Corollary 1. In the noncritical case $\left(P_{Q^{*}}=0\right)$, the solution of the hybrid difference-differential boundary-value problem (2.1)-(2.3) can be represented in the form

$$
\begin{aligned}
& x\left(t, c_{r}\right)=U_{r}(t) c_{r}+V_{r}(t) c_{r}+G\left[\varphi\left(s, \tau_{i}\right) ; \alpha\right](t), \quad c_{r} \in \mathbb{R}^{r}, \\
& y\left(k, c_{r}\right)=Z_{r}(k) c_{r}+W_{r}(k) c_{r}+G\left[\psi\left(k-1, \tau_{i}\right) ; \beta\right](k)
\end{aligned}
$$

with the help of the generalized Green operator of the linear boundary-value problem (2.1)-(2.3)

$$
G\left[\varphi\left(s, \tau_{i}\right) ; \alpha\right](t), \quad t \in \Omega, G\left[\psi\left(k-1, \tau_{i}\right) ; \beta\right](k), \quad k \in \Theta,
$$

where the matrices $X_{r}(t), V_{r}(t), Z_{r}(k)$, and $W_{r}(k)$ specify the general solution of the homogeneous part of the boundary-value problem (2.1)-(2.3); here, $K[\varphi(s, k)](t)$, $K[\psi(k-1)](k)$ is the generalized Green operator of the Cauchy problem (3.1) for system (2.1), (2.2) satisfying the first condition in (2.3). 
Example 2. The problem of construction of the solutions of the hybrid differencedifferential system

$$
x^{\prime}(t)=A x(t)+B y(k)+\varphi(t), \quad y(k+1)=C x(k)+D y(k)+\psi(k)
$$

satisfying the boundary condition

$$
\Delta x\left(\tau_{1}, \tau_{p_{1}}\right):=S_{1} x\left(\tau_{1}-0\right)+b_{1}, \quad \ell x(\cdot)=\alpha \in \mathbb{R}^{2}, \quad \wp y(\cdot)=\beta \in \mathbb{R}^{1},
$$

where

$$
\begin{gathered}
A:=\left(\begin{array}{cc}
0 & 1 \\
-1 & 0
\end{array}\right), \quad B:=\left(\begin{array}{c}
0 \\
1
\end{array}\right), \quad C:=\left(\begin{array}{cc}
1 & 0
\end{array}\right), \quad D:=\left(\begin{array}{l}
1 \\
)
\end{array}\right), \tau_{1}:=\pi, \\
\varphi(t):=\left(\begin{array}{c}
\sin 3 t \\
\cos 3 t
\end{array}\right), \quad \psi(k):=\left(\begin{array}{ll}
\sin k
\end{array}\right), \quad \tau_{2}:=\frac{3 \pi}{2}, \quad \Omega:=[0, \pi[\cup[2 \pi, 3 \pi], \\
\Theta:=\left\{0, \pi, \frac{3 \pi}{2}, 2 \pi, 3 \pi\right\}, \quad S_{\tau_{1}}:=-2\left(\begin{array}{cc}
1 & 0 \\
0 & 1
\end{array}\right), \quad b_{\tau_{1}}:=\left(\begin{array}{l}
1 \\
0
\end{array}\right), \quad \alpha:=\left(\begin{array}{l}
1 \\
2
\end{array}\right), \\
\ell x(\cdot):=x(0)-x(3 \pi), \quad \wp y(\cdot):=y(0)+y(3 \pi), \quad \beta:=\left(\begin{array}{l}
3
\end{array}\right), \quad \tau_{p_{1}}:=2 \pi
\end{gathered}
$$

satisfies the conditions of the proved Corollary 1 .

The matrices

$$
\begin{gathered}
X_{0}(t)=\left(\begin{array}{cc}
\cos t & \sin t \\
-\sin t & \cos t
\end{array}\right), V_{0}(t)=\left(\begin{array}{c}
\sin t \\
-1+\cos t
\end{array}\right), \\
Z(0)=\left(\begin{array}{ll}
0 & 0
\end{array}\right), W(0)=\left(\begin{array}{l}
1
\end{array}\right)
\end{gathered}
$$

specify the general solution

$$
x(t, \zeta, \xi)=X_{0}(t) \zeta+V_{0}(t) \xi, \quad y(0, \zeta, \xi)=Z(0) \zeta+W(0) \xi, \quad \zeta \in \mathbb{R}^{2}, \quad \xi \in \mathbb{R}^{1}
$$

of the homogeneous part of the difference system (4.4) in the interval $[0 ; \pi[$. Similarly, the matrices

$$
Z(\pi)=\left(\begin{array}{ll}
1 & 0
\end{array}\right), W(\pi)=\left(\begin{array}{l}
1
\end{array}\right), Z\left(\frac{3 \pi}{2}\right)=\left(\begin{array}{ll}
0 & 0
\end{array}\right), W\left(\frac{3 \pi}{2}\right)=\left(\begin{array}{l}
3
\end{array}\right)
$$

specify the general solution

$$
y(\pi, \zeta, \xi)=Z(\pi) \zeta+W(\pi) \xi, \quad y\left(\frac{3 \pi}{2}, \zeta, \xi\right)=Z\left(\frac{3 \pi}{2}\right) \zeta+W\left(\frac{3 \pi}{2}\right) \xi
$$

of the homogeneous part of the difference system (4.4) for $k \in\left\{\pi, \frac{3 \pi}{2}\right\}$. The matrices

$$
\begin{gathered}
X_{2 \pi}(t):=\left(\begin{array}{cc}
\cos t & 0 \\
-\sin t & 1
\end{array}\right), V_{2 \pi}(t)=\left(\begin{array}{c}
2 \sin t \\
2 \cos t
\end{array}\right), \\
W\left(\frac{3 \pi}{2}\right)=1, Z\left(\frac{3 \pi}{2}\right)=\left(\begin{array}{ll}
0 & 0
\end{array}\right), Z(2 \pi)=\left(\begin{array}{ll}
0 & -1
\end{array}\right), \\
W(2 \pi)=\left(\begin{array}{ll}
0
\end{array}\right), Z(3 \pi)=\left(\begin{array}{ll}
1 & -1
\end{array}\right), W(3 \pi)=\left(\begin{array}{l}
0
\end{array}\right)
\end{gathered}
$$


specify the general solution

$$
x(t, \zeta, \xi)=X_{2 \pi}(t) \zeta+V_{2 \pi}(t) \xi, \quad y(k, \zeta, \xi)=Z(k) \zeta+W(k) \xi, \quad k \in\{\pi, 3 \pi\}
$$

of the homogeneous part of system $(4.2)$ on the segment $[2 \pi ; 3 \pi]$ satisfying the first boundary condition in (4.3). Since

$$
\mathcal{Q}=\left(\begin{array}{ccc}
2 & 0 & 0 \\
0 & 0 & 2 \\
1 & -1 & 1
\end{array}\right),
$$

we have $P_{Q_{Q}}=P_{Q^{*}}=0$. Therefore, the problem of construction of solutions of the hybrid difference-differential boundary-value problem (4.4), (4.5) corresponds to the noncritical case. Moreover, the unique $(r=0)$ solution

$$
x(t)=G\left[\varphi\left(s, \tau_{i}\right) ; \alpha\right](t), \quad t \in \Omega, \quad y(k)=G\left[\psi\left(k-1, \tau_{i}\right) ; \beta\right](k), \quad k \in \Theta
$$

of the hybrid difference-differential boundary-value problem (4.4), (4.5) can be represented by using the generalized Green operator of the linear boundary-value problem (4.4), (4.5)

$$
\begin{gathered}
G\left[\varphi\left(s, \tau_{i}\right) ; \alpha\right](t)=\left\{\begin{array}{c}
\frac{1}{2}\left(\begin{array}{c}
4 \sin t+\cos t-\cos 3 t \\
-2+4 \cos t-\sin t+\sin 3 t
\end{array}\right), \quad t \in[0, \pi[, \\
\frac{1}{2}\left(\begin{array}{c}
3 \cos t+2 \sin t-\cos 3 t \\
4+2 \cos t-3 \sin t+\sin 3 t
\end{array}\right), \quad t \in[2 \pi, 3 \pi],
\end{array}\right. \\
G[\psi(0) ; \beta](0)=0, \ldots, G[\psi(2 \pi) ; \beta](3 \pi)=0 .
\end{gathered}
$$

Note that scheme of construction and the structure of the generalized Green operator for the hybrid difference-differential boundary-value problem (2.1), (2.2) is independent of degeneracy [18] or nondegeneracy [7,22,24] of the matrices $X_{\tau_{p_{i}}}(t), t \in$ $\Omega$, and $W(k), k \in \Theta$, and the matrix

$$
\Gamma_{\tau_{p_{i}}}(t, k):=\left(\begin{array}{cc}
X_{\tau_{p_{i}}}(t) & V_{\tau_{p_{i}}}(t) \\
Z(k) & W(k)
\end{array}\right), \quad t \in \Omega, \quad k \in \Theta, \quad i=0,1,2, \ldots, q,
$$

specifying the general solution of the homogeneous part of the boundary-value problem $(2.1),(2.2)$. In particular, the matrices $X(t), W(k)$, and $\Gamma_{\tau_{p_{i}}}(t, k)$ in Examples 1 and 2 are nondegenerate.

Example 3. The matrices $X_{\tau_{p_{1}}}(t), W_{\tau_{p_{1}}}(k)$, and $\Gamma_{\tau_{p_{1}}}(t, k)$ degenerate in the problem of construction of solutions of the hybrid difference-differential problem (4.2), (4.3) as a result of the $\operatorname{degenerate} \operatorname{det}\left(I_{2}+S_{1}\right)=0$ pulsed action for $S_{1}:=-I_{2}$.

The matrices $X_{0}(t), V_{0}(t), Z(0)$, and $W(0)$ specifying the general solution $x(t, \zeta, \xi)=X_{0}(t) \zeta+V_{0}(t) \xi, \quad y(0, \zeta, \xi)=Z(0) \zeta+W(0) \xi, \quad \zeta \in \mathbb{R}^{2}, \quad \xi \in \mathbb{R}^{2}$ 
of the homogeneous part of system (4.2) in the interval [0; $\pi$ [ and the matrices

$$
Z(\pi), W(\pi), Z\left(\frac{3 \pi}{2}\right), W\left(\frac{3 \pi}{2}\right)
$$

specifying the general solution

$$
y(\pi, \zeta, \xi)=Z(\pi) \zeta+W(\pi) \xi, \quad y\left(\frac{3 \pi}{2}, \zeta, \xi\right)=Z\left(\frac{3 \pi}{2}\right) \zeta+W\left(\frac{3 \pi}{2}\right) \xi
$$

of the homogeneous part of system (4.2) for $k \in\left\{\pi, \frac{3 \pi}{2}\right\}$ are given in Example 1. Moreover,

$$
\operatorname{det} \Gamma_{0}(0,0)=\operatorname{det} \Gamma_{0}(\pi, \pi)=1 \neq 0 .
$$

Since

$$
\mathcal{Q}=\left(\begin{array}{cccc}
1 & 0 & 0 & 0 \\
0 & 1 & 0 & 0 \\
1 & 0 & 1 & 0 \\
0 & 0 & -1 & 2
\end{array}\right),
$$

we have $P_{Q_{Q}}=P_{Q^{*}}=0$. Therefore, the problem of construction of solutions of the hybrid difference-differential boundary-value problem (4.2), (4.3) corresponds to the noncritical case. Furthermore,

$$
\begin{gathered}
G\left[\varphi\left(s, \tau_{i}\right) ; \alpha\right](t)=\left\{\begin{array}{c}
\frac{1}{2}\left(\begin{array}{c}
\cos t-\cos 3 t \\
-\sin t+\sin 3 t
\end{array}\right), \quad t \in[0, \pi[, \\
\frac{1}{2}\left(\begin{array}{c}
3 \cos t-\cos 3 t-2 \sin t \\
2-2 \cos t-3 \sin t+\sin 3 t
\end{array}\right), \quad t \in[2 \pi, 3 \pi],
\end{array}\right. \\
G[\psi(0) ; \beta](0)=\left(\begin{array}{c}
0 \\
0
\end{array}\right), G\left[\psi\left(\frac{3 \pi}{2}\right) ; \beta\right](2 \pi)=\left(\begin{array}{c}
-1 \\
0
\end{array}\right), \\
G[\psi(2 \pi) ; \beta](3 \pi)=\left(\begin{array}{c}
0 \\
2
\end{array}\right) .
\end{gathered}
$$

Note that, as a result of the degenerate $\operatorname{det}\left(I_{2}+S_{1}\right)=0$ pulsed action, we get the equality det $\Gamma_{0}(2 \pi, 2 \pi)=\operatorname{det} \Gamma_{2 \pi}(3 \pi, 3 \pi)=0$.

Since the set $\Omega \cup \Theta$ is a special case of the set of the "time scale"-type, the accumulated results can be deduced in a similar way $[1,2,4,11]$. The scheme of investigation of hybrid boundary-value problems for systems of difference-differential equations can be applied to the analysis of the problems of bifurcation of solutions of boundary-value problems for difference-differential systems with pulsed action [7], hybrid difference-differential boundary-value problems for systems with pulsed action of a more general form [13,14], matrix difference-differential boundary-value problems for systems with pulsed action [6,12, 15, 16], hybrid difference-integrodifferential boundary-value problems for systems with pulsed action [8,9], and hybrid difference-differential boundary-value problems for systems with pulsed action in abstract spaces [10,17]. 


\section{ACKNOWLEDGEMENT}

This work was financially supported by the State Foundation for Basic Research of Ukraine. Numbers of state Registered 0116U004691 and 0115U003182.

\section{REFERENCES}

[1] R. P. Agarwal and M. Bohner, "Basic calculus on time scales and some of its applications," Results Math., vol. 35, no. 1-2, pp. 3-22, 1999.

[2] R. P. Agarwal, M. Bohner, A. Boichuk, and O. Strakh, "Fredholm boundary-value problems for perturbed systems of dynamic equations on time scales," Math. Meth. Appl. Sci., vol. 38, no. 4, pp. 4178-4186, 2014.

[3] A. D. Andrianov, V. P. Maksimov, and P. M. Simonov, "Boundary-value problems for difference models," Proc. of the Fifth All-Russian Research Conf. Differential Equations and Boundary-Vale Problems, Mathematical Simulation and Boundary-Value Problems, pp. 20-23, 2008.

[4] M. Bohner and A. Peterson, Dynamic Equations on Time Scales. Birkhäuser, Boston, 2001.

[5] A. A. Boichuk, "Boundary-value problems for systems of difference equations," Ukrainian Mathematical Journal, vol. 49, no. 6, pp. 930-934, Jun 1997, doi: 10.1007/BF02513433. [Online]. Available: https://doi.org/10.1007/BF02513433

[6] A. A. Boichuk and S. A. Krivosheya, "A critical periodic boundary value problem for a matrix Riccati equation," Differential Equations, vol. 37, no. 4, pp. 464-471, Apr 2001, doi: 10.1023/A:1019267220924. [Online]. Available: https://doi.org/10.1023/A:1019267220924

[7] A. A. Boichuk and A. M. Samoilenko, Generalized inverse operators and Fredholm boundaryvalue problems. Translated from the Russian by P. V. Malyshev. 2nd edition., 2nd ed. Berlin: De Gruyter, 2016. doi: 10.1515/9783110378443.

[8] O. A. Boichuk and I. A. Holovats'ka, "Boundary-value problems for systems of integrodifferential equations," Journal of Mathematical Sciences, vol. 203, no. 3, pp. 306-321, 2014.

[9] O. A. Boichuk and I. A. Holovats'ka, "Weakly nonlinear systems of integrodifferential equations," Journal of Mathematical Sciences, vol. 201, no. 3, pp. 288-295, 2014.

[10] O. A. Boichuk and A. A. Pokutnyi, "Perturbation theory of operator equations in the Fréchet and Hilbert spaces," Ukrainian Mathematical Journal, vol. 67, no. 9, pp. 1327-1335, Feb 2016, doi: 10.1007/s11253-016-1156-y. [Online]. Available: https://doi.org/10.1007/s11253-016-1156-y

[11] O. A. Boichuk and O. P. Strakh, "Linear Fredholm boundary-value problems for dynamical systems on a time scale," Journal of Mathematical Sciences, vol. 208, no. 5, pp. 487-497, 2015.

[12] S. L. Campbell, Singular Systems of Differential Equations. Pitman Advanced Publ. Program, San Francisco, 1980.

[13] S. M. Chuiko, "Green operator of the boundary-value problem with pulsed action," Differential Equations, vol. 37, no. 8, pp. 1189-1193, Aug 2001, doi: 10.1023/A:1012440123296. [Online]. Available: https://doi.org/10.1023/A:1012440123296

[14] S. M. Chuiko, "Green operator of the boundary-value problem with pulsed action," Doklady Akademii Nauk, vol. 379, no. 2, pp. 170-172, 2001.

[15] S. M. Chuiko, "The Green's operator of a generalized matrix linear differential-algebraic boundary value problem," Siberian Mathematical Journal, vol. 56, no. 4, pp. 752-760, Jul 2015, doi: 10.1134/S0037446615040175. [Online]. Available: https://doi.org/10.1134/S0037446615040175

[16] S. M. Chuiko, "Generalized Green operator of Noetherian boundary-value problem for matrix differential equation," Russian Mathematics, vol. 60, no. 8, pp. 64-73, Aug 2016, doi: 10.3103/S1066369X16080089. [Online]. Available: https://doi.org/10.3103/ S1066369X16080089 
[17] S. M. Chuiko, "Linear boundary-value problem for a matrix differential equation," Differential Equations, vol. 52, no. 11, pp. 1578-1579, 2016.

[18] S. M. Chuiko and E. V. Chuiko, "Generalized Green operator of the cauchy problem with pulsed action," Reports of the National Academy of Sciences of Ukraine, no. 6, pp. 43-47, 1999.

[19] R. Conti, "On ordinary differential equation with interface conditions," Journal Differential Equations, no. vol.4, Issue 1, pp. 4-11, 1968.

[20] A. O. Gel'fand, Calculus of Finite Differences. GIFML, Moscow, 1959.

[21] D. I. Martynyuk, Lectures on the Qualitative Theory of Difference Equations. Naukova Dumka, Kiev, 1972.

[22] A. D. Myshkis and A. M. Samoilenko, "Systems with pushes at given times," Sbornik: Mathematics, vol. 74, no. 2, pp. 202-208, 1967.

[23] G. P. Pelyukh, "On the existence and properties of periodic solutions of discrete difference equations," Ukr. Mat. Zh., vol. 46, no. 10, pp. 1527-1533, Oct 1994, doi: 10.1007/BF01066096. [Online]. Available: https://doi.org/10.1007/BF01066096

[24] A. M. Samoilenko and N. A. Perestyuk, Impulsive Differential Equations. Singapore: World Scientific, 1995.

[25] S. Sčhwabik, "Differential equations with interface conditions," Časopis pro Pěstování Matematiky, vol. 105, pp. 391-410, 1980.

\section{Authors' addresses}

\section{A. Samoilenko}

Institute of Mathematics, Ukrainian National Academy of Sciences, Kiev, Ukraine, Donbass State Pedagogic University, Slavyansk, Ukraine

E-mail address: sam@imath.kiev.ua

\section{A. Boichuk}

Institute of Mathematics, Ukrainian National Academy of Sciences, Kiev, Ukraine, Donbass State Pedagogic University, Slavyansk, Ukraine

E-mail address: boichuk.aa@gmail.com

\section{S. Chuiko}

Institute of Mathematics, Ukrainian National Academy of Sciences, Kiev, Ukraine, Donbass State Pedagogic University, Slavyansk, Ukraine

E-mail address: chujko-slav@inbox.ru 